Voix et Images

\title{
De l'anthropologue au poète : la parole que l'on donne et celle que l'on prend
}

\section{Andrée Mercier}

Volume 12, numéro 1 (34), automne 1986

Québec-Amérique latine

URI : https://id.erudit.org/iderudit/200611ar

DOI : https://doi.org/10.7202/200611ar

Aller au sommaire du numéro

Éditeur(s)

Université du Québec à Montréal

ISSN

0318-9201 (imprimé)

1705-933X (numérique)

Découvrir la revue

\section{Citer cet article}

Mercier, A. (1986). De l'anthropologue au poète : la parole que l'on donne et celle que l'on prend. Voix et Images, 12(1), 113-117.

https://doi.org/10.7202/200611ar d'utilisation que vous pouvez consulter en ligne.

https://apropos.erudit.org/fr/usagers/politique-dutilisation/ 


\title{
De l'anthropologue au poète: la parole que l'on donne et celle que l'on prend
}

\author{
par Andrée Mercier, Université du Québec à Montréal
}

On reconnaît à Pierre Perrault et Rémi Savard un lieu d'exercice de la parole bien distinct: le premier se tient, en amont, à la source d'une parole créatrice (poésie, essai, cinéma), le second, en aval, recueille un discours qu'il s'efforce ensuite de faire signifier (anthropologie). Il n'empêche que l'un et l'autre partagent nettement à la fois une vision du monde et un projet discursif semblables. Pierre Perrault ${ }^{1}$ montre un souci certain de donner la parole/aux gens privés d'écriture (P., p. 243). Comme véritable maître du cinéma-direct et du magnétophone, il condense un discours reçu en autant de caméramages. Et son livre, justement, reprend au fil des pages les "poèmes» d'Alexis Tremblay, de son fils Léopold, des capitaines de goélettes; surgissements de paroles à lire et à écouter: quand i fait beau, prends ton capot/vent du sud, mauvais temps au cul/ cerne à la lune n'a jamais brisé mât d'hune/nordet clair, va coucher avec ta femme à souère (P.,p. 24). Ce rôle de témoin. Rémi Savard ${ }^{2}$ l'assume depuis longtemps déjà, destinataire reconnu de la voix des autres. Pourtant, par désir d'honnêteté, il prend aussi part au discours pour "démythifier" ces récits de l'Autre, trop souvent donnés comme saisis au vol. Le blanc doit révéler sa propre voix et questionner les raisons de [s]a présence sous $[l]$ a tente (S., p. 19), condition nécessaire pour vraiment saisir le sens des paroles de l'Autre. Anthropologues et poètes, Perrault et Savard recherchent l'équilibre entre le privilège de l'écoute et le besoin de l'expression: Mais que peut toute mon admiration pour résoudre leur âme, sinon me procurer la satisfaction d'écrire le poème? (P., p. 286)

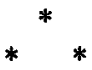

De la parole aux actes réunit douze textes de Perrault parus entre 1966 et 1979. Le recueil se termine donc à la veille de la décennie 80 , bien que les essais aient été revus et mis à jour pour la présente édition. Le parcours de Perrault s'ouvre sur un hommage à la parole des gens d'ici, aux mots qui précèdent l'écriture, à la part d'une humanité brute repoussée par la littérature. Le «Discours sur la parole» et sur les êtres qui l'inspirent, c'est le choix de la voix contre l'image et la forêt de symboles bien rangés sur les rayons de sa bibliothèque (P..p. 9). Pareille préférence, Perrault la réitère tout au long de son ouvrage. Mais cette entreprise ne se limite pas au seul besoin d'un individu, elle vise d'abord et surtout un projet collectif: Parce qu'il y a, ici, quelque part, une terre et mer où la parole a germé, l'humble et cachottière parole épique d'hommes sans écriture et remplis d'admiration pour ce qui donne le plus de pâssion à l'homme; l'amour sous toutes ses formes(P., p. 39). 
Topographe du joualeresque, de l'île aux Coudres à Montréal, en bifurquant par la rivière Coucouchou, Perrault revendique une vision du monde, un territoire non soumis: la Québécoisie pour les siens, l'Amérique pour l'Amérindien. l'Acadie pour les Acadiens... Si le géographe ne peut que constater la conquête et confirmer les occupations ( $\mathrm{P} ., \mathrm{p} .51)$, le détecteur de paroles, lui, déplace les bornes. Il nomme l'espace tel que voulu et rêvé, mais il lui faut le nommer avec du vécu (P., p. 48). Sinon, telle l'Acadie, il n'en découlera rien d'autre qu'une géographie de l'âme (P., p. 213). En fait, chaque essai affirme une parole et exige l'acte: Le poème n'est que beau. Il arrive que l'action soit sublime (P., p. 361). Car, à n'être que locataire du langage, détenteur d'une parole folklorique et marginale, le peuple de la Québécoisie reste coincé dans la clandestinité. Envisager le passage de la parole aux actes, c'est voir venir le temps de la maittrise et la fin de la fiction aphrodisiaque (la bière de Molson, les quatorze soleils d'Air Canada):

Et alors nous cesserons de nous mettre au musée, de conserver le patrimoine, de racheter nos armoires à l'ennemi. Nous recommencerons, ce jour-là, à produire du patrimoine. Nous nous tiendrons responsables de la courbe de nos toits. Nous confierons à un armoirier sublime le soin de construire un stade monumental pour affirmer nos maîtrises et notre orgueil.

Toutefois, la logique même du recueil n'aboutit pas à l'acte, elle s'en arrête tout au seuil. En effet, ce livre d'espoir, de foi et d'exigence préfère s'achever sur une ultime interrogation et laisser aux lecteurs. et lectrices le soin de supposer la réponse. L'auteur n'a-t-il pas cru bon de taire tout épilogue et ce, malgré la mise à jour récente des textes?

Aurons-nous le courage de nous mépriser nous-mêmes au point de refuser d'envisager seulement l'hypothèse d'une libération, de refuser le simple combat des négociations?

Aurons-nous le courage de laisser échapper une aussi belle embelle? L'indécence de ne pas, enfin, passer de la parole aux actes?

Et j’en aurais à mon tour la mort dans l'âme!!!

Ce parcours étendu sur deux décennies ne forme d'ailleurs pas une courbe ascendante et continue vers l'espoir. Les propos de Pierre Perrault oscillent toujours entre l'espérance (Elle [la parole] finira bien par déloger Allo Police et la Molson des esprits P., p. 391) et le doute (Cependant je m'inquiète de l'épuisement de la parole et de son désistement P., p. 318). D'un texte à l'autre, quand ce n'est à l'intérieur d'un même essai, il y a tiraillement d'un désir et d'une réalité. Du sommet euphorique que constitue la «Lettre du Québec" au point final inquiet de "Â propos de la mort dans l'âme», le discours de Perrault craint sans cesse la capitulation de la parole ${ }^{3}$. 
Rémi Savard choisit aussi la parole, la voix des autres, celle du peuple algonquien. C'est autour de Tshakapesh, personnage éminemment commenté par les destinataires blancs, qu'il tente de rétablir le primitif, à titre de sujet parlant, dont la rigueur du discours ne le cède en rien à celle du discours savant porté sur lui (S., p. 68). Si Perrault se méfie de la belle ordonnance des symboles d'une littérature, Savard recherche dans la parole la cohérence qu'on lui a longtemps refusée. Au moins le structuralisme peut-il aider à la reconnaissance d'une logique concrète des "mythes»" 4 . En fait, le projet de Savard en est aussi un de réhabilitation de la parole. Le "mythe» recouvre, au sein des théories explicatives savantes, une forme élémentaire de la pensée humaine. Pareille désignation ramène le conteur à une sorte d'amplificateur qui rapporte à peu près mécaniquement, et sans trop le comprendre, ce résidu passif d'un passé achevé. Concept rassurant, puisqu'il confirme l'extinction prochaine de sociétés statiques. Le "mythe" ne s'en avère pas moins un discours pertinent sur la situation actuelle des autochtones. La Voix des autres se donne justement pour but de prouver trois aspects actifs du récit "mythique»: 1. son inscription dans des circonstances particulières de transmission souvent traumatisantes, la colonisation par exemple 2. une signification non seulement en regard d'un passé lointain mais surtout d'un présent 3. une organisation interne nettement rigoureuse et ce, jusqu'au niveau des groupes de transformations (l'ensemble des variantes).

L'ouvrage accorde une importance variable à chacun des objets. Toutefois, la balance penche évidemment du côté de la voix des autres: la dernière partie du texte regroupe quelques 49 variantes du récit de Tshakapesh (recueillies entre 1637 et 1978); près de la moitié du livre recouvre ainsi la parole algonquienne. C'est dire que dans les faits, l'ouvrage n'infirme pas le désir de préserver la voix de l'Autre et circonscrit la démonstration des objectifs "savants" à un nombre limité de pages. Soucieux de rétablir le véritable contexte d'énonciation du "mythe", Savard choisit de se mettre en place, dans un court prologue, en tant que destinataire blanc du récit: J'étais chez les Paukuaushipiunnut. Sur la rive ouest du fjord recevant les eaux de la rivière Saint-Augustin (S., p. 13). Dans le même but, s'ajoutent quelques extraits du journal de Louis Jolliet, venu là trois siècles plus tôt, et la présence de Bing Crosby, projeté une fois de plus dans Say one for me. Deux rencontres, deux temps distincts, d'où Tshakapesh sort pourtant vainqueur: Il était très fort ce Tshakapesh, dira le jeune Pien ce jeudi, 11 février 1971. Après cette brève mise en situation, Savard interroge cette fois l'enjeu de pareils récits «mythiques" pour le discours savant. Tshakapesh, avant de prendre sens au coeur du discours algonquien, doit comprendre sa fonction anthropologique. Sait-on, par exemple, que le dossier québécois sur la nature des formes précolombiennes d'organisation socio-territoriale (S., p. 60) - chez les différentes bandes recensées par F.G. Speck - alimentera un fort débat sur le caractère naturel ou non de la propriété privée? Débat où ethnologues soviétiques mêmes s'opposèrent à une interprétation favorable au capitalisme américain? Ce n'est qu'une fois bien reconnues les fonctions institutionnelles de l'anthropologie - travail de décapage du discours savant - , que Savard pénètre ensuite dans le discours algonquien. Par la voix autorisée de Penashue 
Pepine, l'histoire de Tshakapesh apparait alors. Pour atteindre la signification et la cohérence du "mythe", Savard adoptera une perspective structurale. Ce choix se fait d'abord contre la tendance de plusieurs analystes à sortir les éléments sémantiques de l'ordre du récit. Un exemple, celui du "super-ours" dont la trompe énigmatique fit supposer, entre autres, la contemporanéité du mammouth américain et des ancêtres indiens. La querelle d'hypothèses montre assez l'idée que l'on propose du "mythe": un effort plus ou moins maladroit, de la part de gens mal partagés sur le plan de la rigueur, en vue de relater des faits réels (S., p. 114). Quant à l'allure éléphantesque du super-ours, au-delà d'indices paléontologiques, si elle provenait plus simplement de la relation d'opposition que cette image entretient avec une autre située à la fin du récit: la musaraigne masquées, c'est-à-dire le plus petit des mammifères terrestres du Nord-Est américain (S., p. 117)? La suite de l'étude illustre à d'autres niveaux la cohérence sémantique du "mythe" de Tshakapesh en ayant recours à l'ensemble des variantes. La parole se soumet toujours à l'ordonnance, aussi bien à l'intérieur d'un récit que dans le groupe de transformations.

En regard du Rire précolombien dans le Québec d'aujourd'hui ${ }^{6}$, Savard a su mettre en lumière la rigueur du discours algonquien en jeu dans un corpus encore plus considérable (49 variantes). Il montre aussi une constance dans ses exigences de rétablir la voix des autres, de la mettre clairement en situation coloniale contemporaine. Toutefois, le «mythe» de Kamikwakushit (étudié dans le Rire...) convainquait davantage de sa signification et de son rôle actuels dans le mode de vie montagnais. Il ressort que, si le dernier ouvrage de Savard éclaire la fonction du mythe entretenue par une anthropologie institutionnelle, il formalise peu les circonstances qui déterminent l'énonciation de Tshakapesh. L'analyse du «mythe» a-t-elle vraiment démontré la capacité de réflexion, d'affirmation et de résistance face aux conditions créées par la situation coloniale (S., p. 137)?

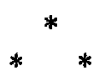

À leur façon respective, Savard et Perrault construisent une vision du monde semblable, puisque fondée sur des choix communs. Leurs pratiques discursives visent fondamentalement une réhabilitation de la parole analphabète contre la mainmise de l'écriture. Pareille entreprise en est une d'abord de dénonciation. Savard combat l'effort de «mythification» de la voix des autres, stratégie institutionnelle qui mène à magnifier sa propre culture au détriment du discours inopportun et impertinent de l'Autre. Sa position devant la face cachée de l'anthropologie est nettement définie:

L'hypothèse sur laquelle repose le présent ouvrage est que le "mythe», en tant que mode d'expression privilégié par les chasseurs-cueilleurs du Québec, leur a servi d'outil de réflexion, d'affirmation et de résistance face aux conditions créées par la situation coloniale. 
De son côté, Perrault opère le même choix et se met à l'écoute des gens d'ici à peine objet d'anthropologie (P., p. 11): J'ai quasi renoncé aux Écritures, à toutes les paroles enfouies dans les bandelettes de l'écriture. Je m'instruis de paroles vivantes, ardentes et terrestres (P., p. 144). Son action, il la mène aussi contre une mythification par la mise en fiction d'un patrimoine, la perte de nos maîtrises, au profit des fictions aphrodisiaques de Molson, de Kraft... Témoins donc, et promoteurs de la voix, tous deux revendiquent le discours pertinent et subversif de qui refuse la mort. Perrault y voit la force du poème, Savard lui trouve la cohérence du discours savant. Chacun, dans son lieu singulier, prend la parole. L'un et l'autre pour mieux la donner.

Pourtant, ce choix ne peut préserver la voix dans toute son intégrité et doit, ne serait-ce que pour la diffuser, retourner à l'écriture. Nécessité de discourir sur des mots auxquels manquent l'élégance d'une langue classique, le geste, les vibrations de la voix, le corps et les yeux (S., p. 81); l'intonation... la tissure même de la voix... l'odeur du souffle... la dent noircie qui sait rire à pleine gorge... la rondeur du palais qui veloute les mots (P., p. 28).

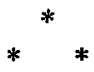

1. Pierre Perrault, De la parole aux actes, Montréal, l'Hexagone, coll. «Essais», 1985. 435 p. Les références à l'ouvrage: (P.. p. X).

2. Rémi Savard, la Voix des autres, Montréal, l'Hexagone, coll. «Positions anthropologiques", 1985, 351 p. Les références à l'ouvrage: (S.. p. x).

3. Pour un aperçu critique de la démarche de P. Perrault cinéaste, les Traces du rêve de Jean-Daniel Lafond propose un portrait attachant et lucide. Film produit et distribué par l'ONF, 1986.

4. Devant l'ambiguïté péjorative du mot mythe, nous reprendrons l'habitude typographique de Savard: «mythe», «mythique».

5. Le super-ours, mis en rapport avec les autres personnages du récit, représente le plus gros des mammifères terrestres du territoire. De plus, sa trompe semble le rapprocher d'un autre animal fouisseur du texte, la musaraigne masquée. (S., p. 117 et 124-125).

6. Rémi Savard, le Rire précolombien dans le Québec d'aujourd'hui, Montréal, l'Hexagone/ Parti pris, 1977, 157 p.

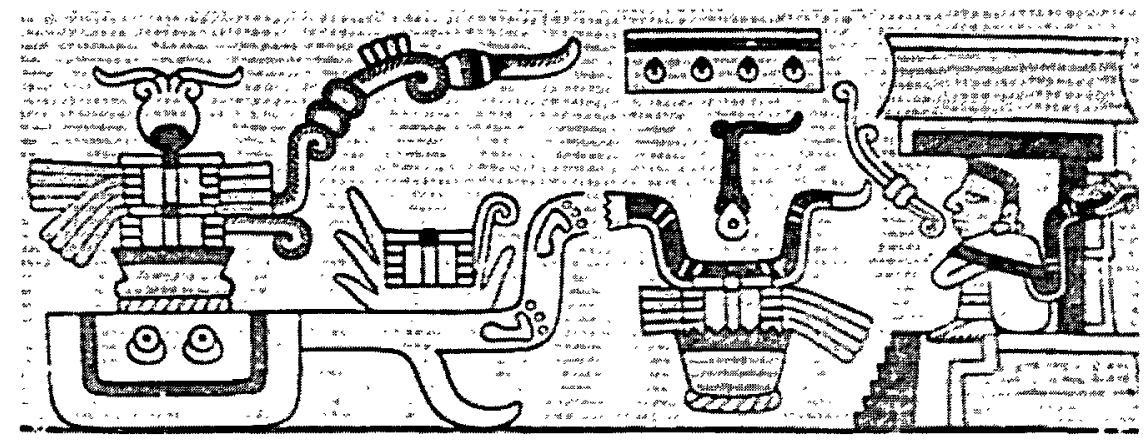

